Research Article

\title{
Coupling Mechanism of Rotating Casing Effect and Impeller Structure of Roto-Jet Pump
}

\author{
Qi Huang $\mathbb{D}^{1},{ }^{1}$ Zailun Liu $\mathbb{D}^{1},{ }^{1}$ Xiaobing Wang, ${ }^{2}$ Qifei Li, ${ }^{1}$ and Hui Quan ${ }^{1}$ \\ ${ }^{1}$ School of Energy and Power Engineering, Lanzhou University of Technology, Lanzhou 730050, China \\ ${ }^{2}$ Lanzhou Vacuum Equipment Co. Ltd. Huayu Branch, Lanzhou 730050, China \\ Correspondence should be addressed to Zailun Liu; liuzl88@sina.com
}

Received 19 August 2020; Revised 30 September 2020; Accepted 6 October 2020; Published 24 October 2020

Academic Editor: Yong Zhu

Copyright (c) 2020 Qi Huang et al. This is an open access article distributed under the Creative Commons Attribution License, which permits unrestricted use, distribution, and reproduction in any medium, provided the original work is properly cited.

An increase in internal pressure of a Roto-Jet pump is due to combined action of its impeller and rotating casing. Internal pressure of the pump chamber was determined according to outlet pressure of the impeller, and the influence of the rotary casing effect was ignored. To study the combined action mechanism of the rotating casing effect and impeller structure on the Roto-Jet pump, we used the open test bed of the Roto-Jet pump and four model pumps with impellers of different structures as research objects. We also conducted a comprehensive experimental study on the coupling mechanism between the rotating casing effect and impeller structure. Numerical calculation was performed to avoid the assumption of isotropic eddy viscosity, Reynolds stress linear pressure-strain model is selected, and the numerical calculation results are compared with the experimental results to verify its credibility. The results show that the rotating casing effect has multiple functions to reduce the friction loss of the disc, improve the pressure distribution inside the rotating casing, and increase the pump head. All scheme, pressure, and velocity fluctuations occur in the upstream and wake regions of the collecting pipe and the energy loss is concentrated in the upstream region. The difference in velocity distribution inside the collecting pipe is small and negligible. As long as the impeller and rotating casing continue to rotate synchronously, the liquid shear velocity at the same coordinate position of each scheme remains unchanged, and the liquid rotation angular velocity in the rotating casing is approximately $75 \%$ of the rotating casing which conforms to the rigid motion law. In the same scheme, the coefficient of uneven velocity inside the rotating casing gradually increases along the radial direction. The closer to the axis, the faster is the decrease of the peak tangential velocity and the velocity tends to be uniform. The size, shape, and position of the vortex core inside the rotating casing change constantly with various schemes. The distribution of vortex cores varies under each scheme. The front cover and rotating casing have a serious effect on the vortex core. The extremely poor analysis of the test results shows that the performance of the Roto-Jet pump is better when the closed impeller is rotated in synchronisation with the rotating casing. The advantages and disadvantages of each blade type can be determined according to the situation. The research results can exhibit the influence mechanism of the Roto-Jet pump shell effect. Selection of an impeller structure provides a reference.

\section{Introduction}

A Roto-Jet pump is a type of small flow, high head, low specific speed pump [1] and its flow parts differ from those of ordinary centrifugal pumps. The Roto-Jet pump impeller and rotary casing rotate synchronously to reduce disc friction loss. The efficiency of low specific speed pump is higher than that of an ordinary centrifugal pump [2]. At present, research on Roto-Jet pumps mainly focuses on theoretical and numerical calculations and optimised modification designs. For example, Tanasawa and Miyasaka $[3,4]$ provide theoretical calculation formulas for dynamic pressure, static pressure, total pressure, and flow rate of Roto-Jet pumps. This formula shows that its internal pressure distribution is only related to the weight of the medium, diameter of the rotating casing, and speed. Yang [5] proposed the optimisation design method of the Roto-Jet pump impeller and design principles of hydraulic parameters. Zhu et al. [6] installed a baffle inside the collecting pipe to slightly reduce the radial force on the collecting pipe. 
Wang et al. [7] used the standard turbulence model and SIMPLEC algorithm to summarise the flow of the internal flow channel of the impeller; Liu et al. [8] used numerical simulation methods to optimise and modify the chamber structure of the jet pump. Komaki et al. $[9,10]$ believe that the flow in the rotating casing is similar to the forced vortex type. The larger the cross-sectional area of the collecting pipe channel, the greater the head and flow rate and the higher the efficiency. Komaki also confirmed that the secondary flow is behind the collecting pipe. The wake area flows towards the centre of rotation. Sagara et al. [11] showed that the short-tolong axis ratio of 0.25 elliptical collecting pipe has the highest efficiency near 1.2 times the rated flow through the transitional change of the inner diameter shape of the collecting pipe. Hattori and Kato $[12,13]$ studied the external characteristics and internal flow of the Roto-Jet pump and the shape of the inlet of the collecting pipe to reduce the frictional hydraulic loss of the collecting pipe. The pressure in the Roto-Jet pump chamber has been determined according to the outlet pressure of the impeller [14], ignoring the influence of the rotating casing on the pressure field; the head of the Roto-Jet pump is believed to be mainly derived from the impeller and this simplified processing has certain limitations. As a consensus, the synchronous rotation of the rotating casing and impeller can reduce the friction loss of the disc and improve the pump efficiency. However, the other influence mechanism of the rotating casing effect on the pump performance has yet to be further studied. The Roto-Jet pump performs work on the fluid by rotating the impeller and rotating casing, so that the fluid forms a circulating flow in the pump chamber. The influence mechanism of the rotating casing effect on the performance of the Roto-Jet pump is not yet sufficient and, in terms of numerical calculations and experiments, it is often only a single structural type and other structural types are fixed at a time. The coupling of multiple factors on the performance of the jet pump is rarely considered. The Roto-Jet pump is equipped with multiple impeller structure types at the same time and a comprehensive test is conducted with the rotating casing effect. The rotating casing effect and influence mechanism of the impeller structure type on the internal and external characteristics of the Roto-Jet pump are studied in depth. Optimal matching scheme among jet pump flow, head, and efficiency was applied. According to the internal flow law of the Roto-Jet pump, examining the nature of the influence of the rotating casing and impeller structure, improving the performance of the Roto-Jet pump, and expanding the operating range are important tasks.

\section{Establishment of Calculation Model}

2.1. Numerical Calculation Model. We consider the test Roto-Jet pump as the object to establish the numerical calculation model. The Roto-Jet pump working medium is clean water with rated flow $Q_{0}=7.5 \mathrm{~m}^{3} / \mathrm{h}$, rated head $H_{0}=80 \mathrm{~m}$, rated speed $n_{0}=2900 \mathrm{r} / \mathrm{min}$, and rated efficiency $\eta_{0}=25 \%$. NPSHr must be equal to $1.8 \mathrm{~m}$. The main geometric parameters of the test pump are shown in Table 1.
TABLE 1: Geometrical parameters of the test pump.

\begin{tabular}{lcc}
\hline Name & Parameter & Value \\
\hline Impeller inlet diameter & $D_{1} / \mathrm{mm}$ & 50 \\
Impeller outer diameter & $D_{2} / \mathrm{mm}$ & 249 \\
Blade outlet width & $b_{2} / \mathrm{mm}$ & 6 \\
Number of blades & $z$ & 5 \\
Diameter of rotating casing & $D_{3} / \mathrm{mm}$ & 310 \\
Width of rotating casing & $b_{3} / \mathrm{mm}$ & 77 \\
\hline
\end{tabular}

To show the influence of the impeller structure and rotating casing effect on the internal flow state, the blade type selects the backward curved/straight blade, the impeller structure selects the closed/semiopen type, and the rotating casing is divided into rotating and nonrotating. The modelling method is as follows. Firstly, SolidWorks is used to build a $1: 1$ model according to the geometric parameters of the test pump, which is recorded as model 1. For comparison with the test results, we keep the other structural dimensions of model 1 unchanged and remove the front cover to form a semiopen impeller, which is recorded as model 3. Secondly, keeping the impeller channel of model 1 and the diameter of the blade inlet and outlet unchanged, using straight blades instead of backward curved blades, we record it as model 5 and remove the front cover of model 5 as model 7. All model calculation domains are shown in Figure 1.

2.2. Meshing. The calculation model adopts ICEM grid division. The water model is divided into four parts: inlet extension, impeller, rotating casing, and collecting pipe. The total number of grids generated in model 1 is 7,911,042 and the number of nodes is 1,561,926 (the rest of the models are slightly different). The key components in the model are locally encrypted to improve the calculation accuracy. The local grid is shown in Figure 2(a). Grid independence test verification is conducted at rated operating point and shown in Figure 2(b). When the number of grids is greater than 6 million, the correlation of head is within $0.5 \%$ and it can be considered that the grid has no effect on the calculation results.

\subsection{Numerical Simulation Methods and Boundary} Conditions. The internal flow of the Roto-Jet pump is a complex 3D incompressible flow that rotates around a fixed axis. According to the characteristics of model l, to cope with the flow characteristics of high strain rate and large degree of curvature of the streamline in the pump chamber $[15,16]$, we have to improve the accuracy of the model in the case of large curvature and avoid the assumption of isotropic vortex viscosity. The numerical calculation choice has a higher prediction for complex flow accurate Reynolds stress linear pressure-strain model. The pump inlet adopts mass flow inlet, no-slip wall hypothesis, and standard wall function method and adopts a combination of dynamic and static dual reference systems to deal with the problem of liquid flow in the manifold and rotor chamber. The flow surface roughness is $25 \mathrm{um}$; the rotating casing and impeller adopt the rotating coordinate system. The speed is $2900 \mathrm{r} / \mathrm{min}$, the 


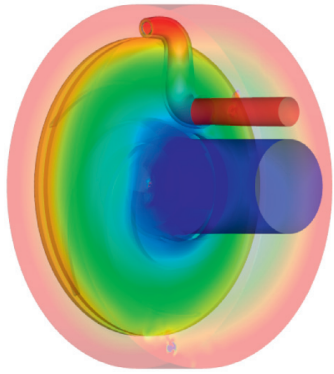

(a)

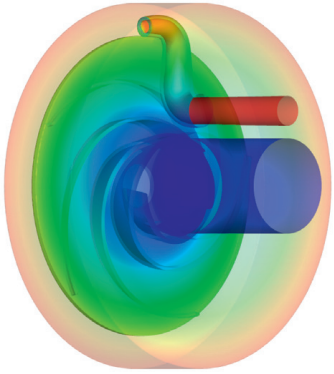

(b)

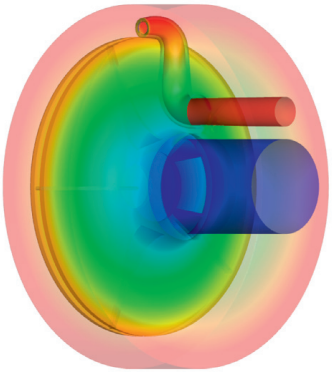

(c)

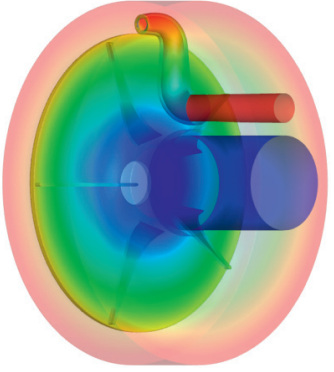

(d)

Figure 1: Numerical calculation model of Roto-Jet pump. (a) Model 1. (b) Model 3. (c) Model 5. (d) Model 7.

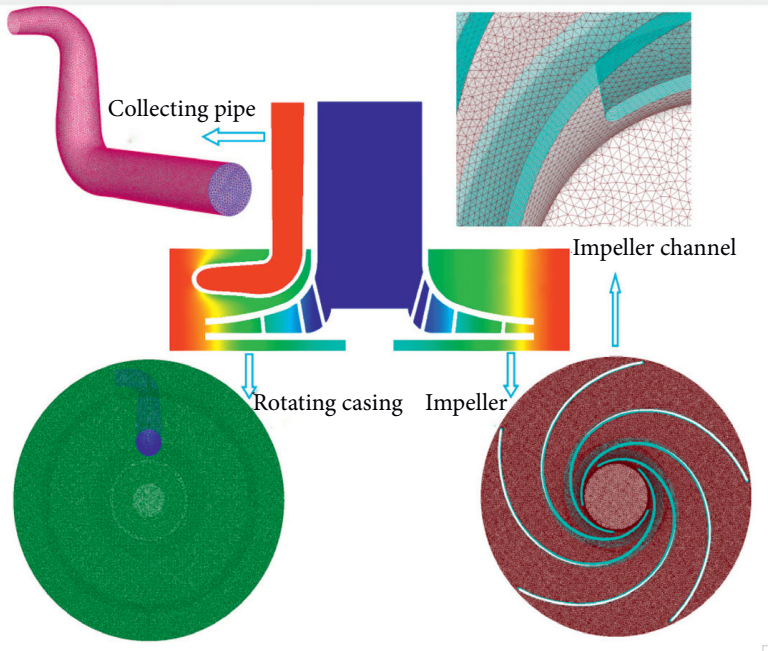

(a)

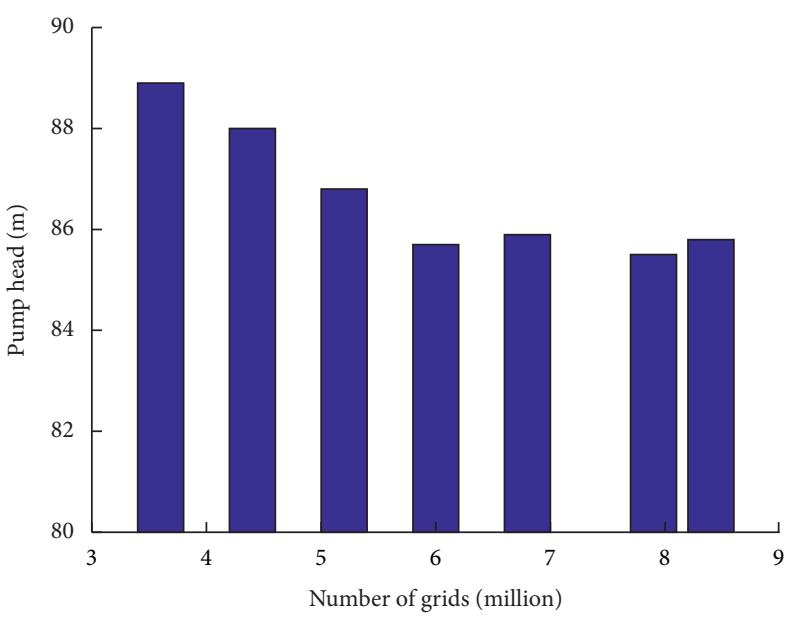

(b)

Figure 2: Grids of Roto-Jet pump and grid independence verification. (a) Grids of Roto-Jet pump. (b) Grid independence verification.

collecting pipe adopts the static coordinate system, the outlet adopts the free flow boundary condition, the pressure and velocity coupling SIMPLE algorithm, the standard format pressure subrelaxation term, momentum, turbulent kinetic energy, and dissipation, and the rates are all second-order upside-down discrete difference equations. Fluent 16.0 is used to calculate the constant value and the residual $10^{-5}$ is taken as one of the criteria for judging the degree of convergence of the calculation.

\section{Experimental Design, Data Extraction, and Reliability Verification}

The Roto-Jet pump is tested and the numerical calculation is performed on the same structure model 1. The test data are compared with the numerical calculation data to check whether the numerical calculation data are reliable. The external characteristic test system is shown in Figure 3(a). The internal pressure test is shown in Figure 3(b). A piezometer tube is installed inside the rotating casing. The piezometer and collecting tubes are in a $180^{\circ}$ symmetrical arrangement. Seven pressure-measuring holes are on the piezometer tube. We read the pressure in the chamber by opening one pressure-measuring hole and closing six of these holes. The radii $r$ between the centre of the pressure measuring hole and axis of the pump are 72, 82.5, 93, 103.5, $114,124.5$, and $135 \mathrm{~mm}$.

3.1. Experimental Design. This study focuses on the coupling mechanism of Roto-Jet pump impeller structure and rotating casing effect and introduces three influencing factors: blade type, impeller front cover, and rotating casing. Each factor has two levels. $A, B$, and $C$ represent three different influencing factors. A comprehensive test design was selected based on the actual situation of the Roto-Jet pump. The eight structural configuration schemes are shown in Table 2 . In this table, $A_{1}$ represents the blade type as a backward curved blade, $A_{2}$ represents the blade type as a straight blade, $B_{1}$ represents a closed impeller with a front cover, $B_{2}$ represents a semiopen impeller without a front cover, $C_{1}$ represents the rotation of the rotating casing, and $C_{2}$ represents that the rotating casing does not rotate. 


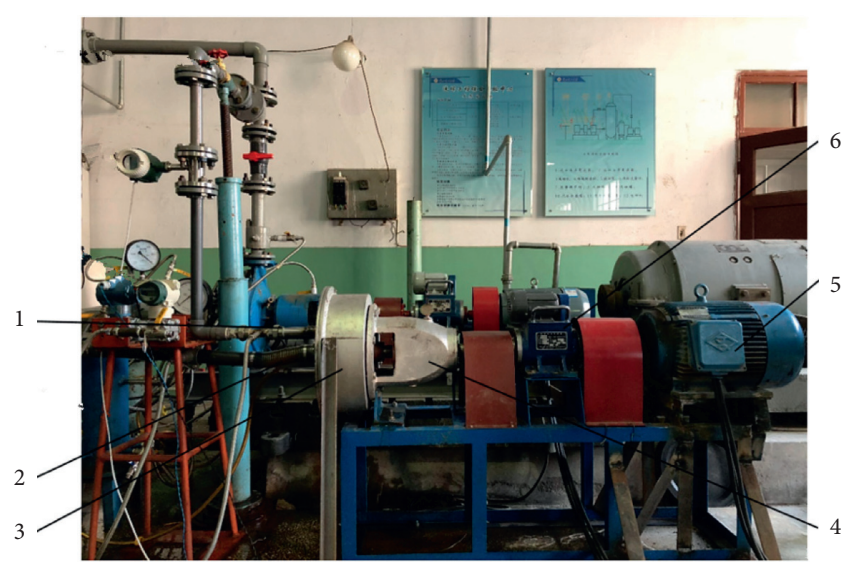

(a)

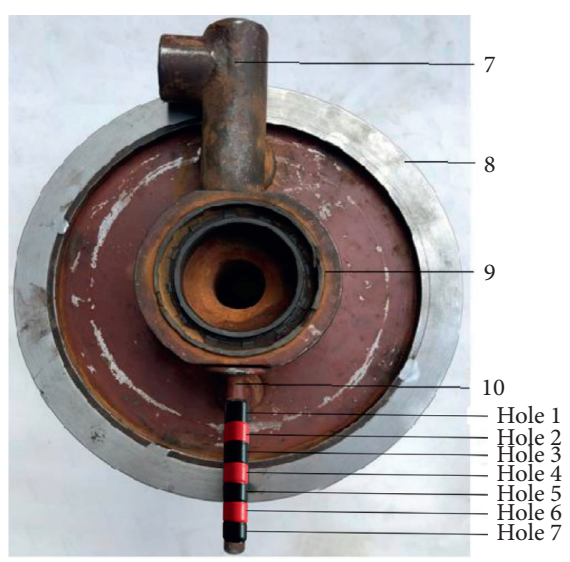

(b)

Figure 3: Test system. (a) Performance test bench for Roto-Jet pump. (b) Collecting pipe and piezometer tube. 1: collecting pipe outlet; 2: impeller inlet; 3: Roto-Jet pump; 4: suspension; 5: motor; 6: torquemeter; 7: collecting pipe; 8: fixed plate; 9: framework oil seal; 10: piezometer tube.

TABLE 2: Configuration scheme of model structure.

\begin{tabular}{lccc}
\hline Test scheme & \multicolumn{3}{c}{ Factor } \\
& Blade type & Front cover & Rotating casing \\
\hline$S_{1}$ & $A_{1}$ & $B_{1}$ & $C_{1}$ \\
$S_{2}$ & $A_{1}$ & $B_{1}$ & $C_{2}$ \\
$S_{3}$ & $A_{1}$ & $B_{2}$ & $C_{1}$ \\
$S_{4}$ & $A_{1}$ & $B_{2}$ & $C_{2}$ \\
$S_{5}$ & $A_{2}$ & $B_{1}$ & $C_{1}$ \\
$S_{6}$ & $A_{2}$ & $B_{1}$ & $C_{2}$ \\
$S_{7}$ & $A_{2}$ & $B_{2}$ & $C_{1}$ \\
$S_{8}$ & $A_{2}$ & $B_{2}$ & $C_{2}$ \\
\hline
\end{tabular}

3.2. Experimental Data Extraction. The collecting pipe is critical to the performance of the Roto-Jet pump and liquid flow state in the rotary casing. The impeller is the main work component inside the Roto-Jet pump. Therefore, the model selects the characteristic axis section $Z_{1}$ passing through the centre point of the inlet of the collecting pipe and perpendicular to the centre line of the pump shaft and the characteristic axis section $Z_{2}$ passing through the centre point of the impeller outlet and perpendicular to the centre line of the pump shaft. The distance between sections $Z_{1}$ and $Z_{2}$ is $40 \mathrm{~mm}$, as shown in Figure 4 . The $Z_{1}$ characteristic section of the calculation model corresponds to the characteristic axis section of the test pump pressure measuring point. We select seven concentric circles with radii of $135,124.5,114,103.5,93$, 82.5 , and $72 \mathrm{~mm}$ on the $Z_{1}$ axis section and mark them as $\mathrm{O}_{1}, \mathrm{O}_{2}, \mathrm{O}_{3}, \mathrm{O}_{4}, \mathrm{O}_{5}, \mathrm{O}_{6}$, and $\mathrm{O}_{7}$. The seven curves pass through the seven pressure measurement points and the $O_{1}$ curve passes through the central area of the inlet of the collecting pipe to avoid interference between the internal pressure of the collecting pipe and the internal pressure of the rotating casing when reading the data. The $\mathrm{O}_{1} \sim \mathrm{O}_{7}$ curve is hereby truncated and the cut-off lines are marked as $L_{1}$ and $L_{2}$. The angle is $32^{\circ}$ between the cut-off lines $L_{1}$ and $L_{2}$ at the beginning and end of the flow.
3.3. Test Reliability Verification. For the backward-curved closed impeller of scheme $S_{1}$, we conduct tests and numerical calculations at rated operating point. The internal pressure and external characteristic curves of the Roto-Jet pump were obtained as shown in Figure 5.

By analysing Figure 5, we can observe that as the flow rate gradually changes from small to large, errors occur between the test head $H_{t}$ and numerical simulation head $H_{1}$ and between test efficiency $\eta_{t}$ and numerical simulation efficiency $\eta_{1}$ of the Roto-Jet pump. After calculation, the test value of the head at the rated working condition is $83.6 \mathrm{~m}$ and the simulation value is $82.7 \mathrm{~m}$, the average error of the head is $2.91 \%$, and the maximum error is $5.4 \%$. The test value of the rated operating point efficiency is $25.8 \%$ and the simulated value is $25.6 \%$, the average error of efficiency is $3.03 \%$, and the maximum error is $5.8 \%$, all of which are less than $6 \%$, which proves that the numerical method is reliable for the pump research. According to the pressure test data of the pressure measuring points $H_{t 1} \sim H_{t 7}$, the pressure of each pressure measuring point decreases linearly with the increase of the flow rate and is distributed in parallel lines. As the inlet centre point of the collecting pipe and pressure measuring point 1 are located on the same circumferential curve $O_{1}$, subsequent studies have shown that the internal pressure contours of the rotating casing are close to concentric circles; the curve $H_{t 1}$ reflects the relationship between the pressure at the inlet of the collecting pipe and the flow rate. Both $H_{t}$ and $H_{t 1}$ decrease with the increase of the flow rate and the deviation between the two increases with the increase of the flow rate. The friction loss inside the collecting pipe is proportional to the square of the flow rate and the impact loss is proportional to the square of the deviation from the rated flow rate [17]. Large flow conditions have greater friction loss and impact loss, leading to an increase in the deviation between the two and a significant drop in the head. At the same flow rate, the data of each pressure measurement point shows a parabolic increase along the radial direction. The larger the radius is, the more obvious the effect of the rotating casing rotation is. The liquid molecules are dragged 


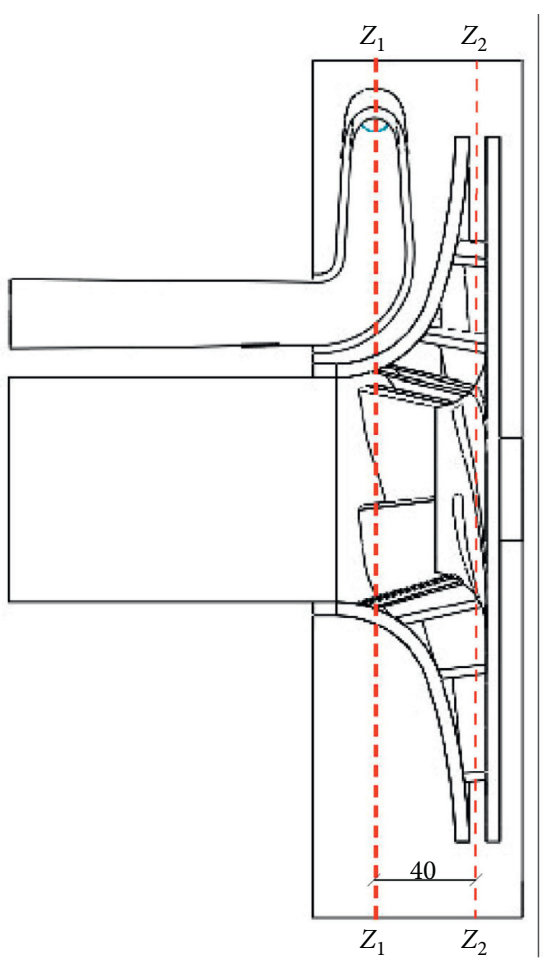

(a)

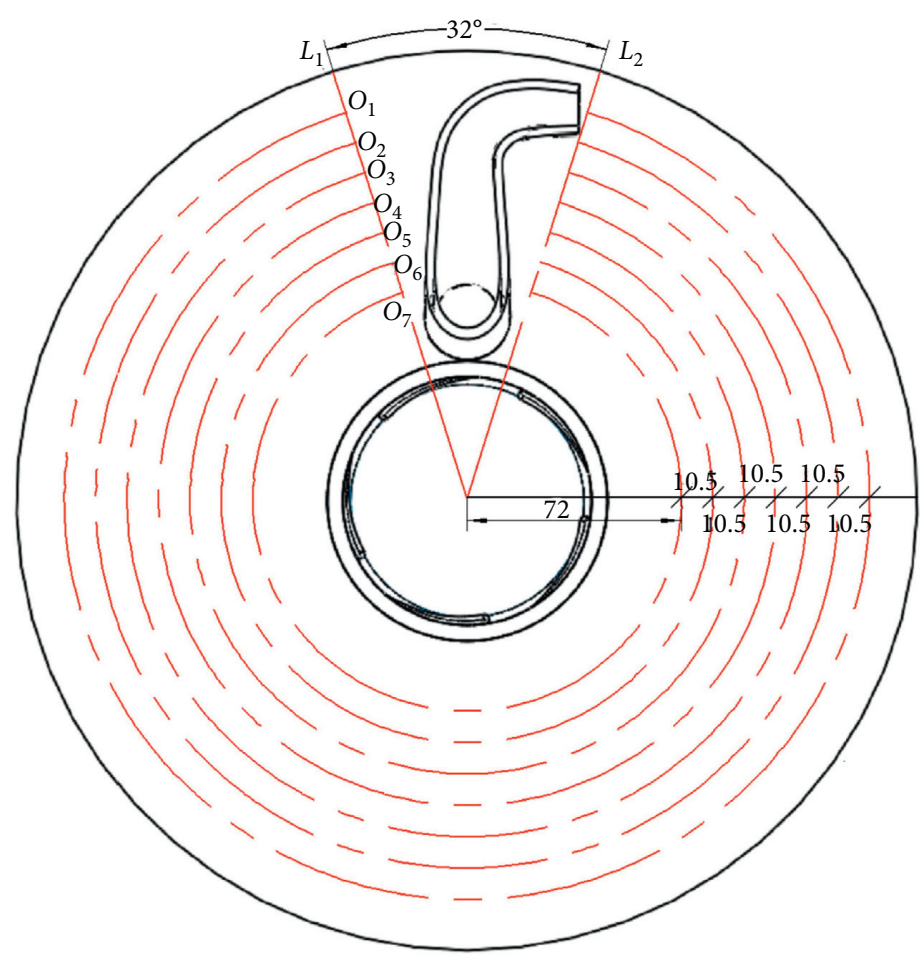

(b)

FIGURE 4: Schematic of characteristic section.

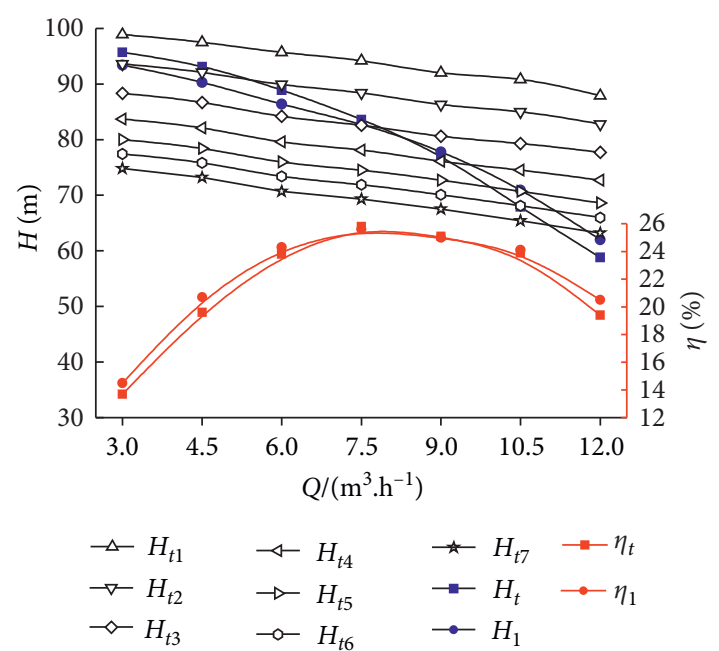

FIGURE 5: Internal pressure and performance curve.

by the inner wall of the rotating casing to make a rotary shearing motion; the energy transfer causes an increase in the pressure inside the rotating casing. The rotating casing effect has the dual function of reducing the friction loss of the disc and increasing the energy of the liquid. For the other schemes designed in this experiment, numerical simulations are performed to save time and resources.

\section{Analysis of Test Results at Rated Operating Conditions}

4.1. Pressure Distribution in Rotating Casing. Numerical calculations are conducted for schemes $S_{1}-S_{8}$ at the rated operating point. The pressure distribution of the $Z_{1}$ axial section is shown in Figure 6.

Figure 6 shows that the liquid pressure in the chamber increases with the radius in the radial direction in the eight schemes. In the same scheme, the pressure of the liquid with the same radius is basically unchanged, and the distribution of isobars is approximately concentric, indicating that the axial velocity of the liquid in the chamber is small and the liquid flow in the chamber can be simplified as a plane flow. A slight height difference is observed in the isobaric lines near the collecting pipe. When the radius is the same, the pressure in the upstream area of the collecting pipe is higher than that in the wake area, and the high-speed rotating fluid collides and gathers on the upstream surface of the collecting pipe, causing the pressure in this area to rise. The comparison showed that because schemes $S_{5}, S_{6}, S_{7}$, and $S_{8}$ use straight-blade impellers, the internal pressures are higher than those of schemes $S_{1}, S_{2}, S_{3}$, and $S_{4}$ which use backward curved impellers in the same working condition. This condition is consistent with the theoretical analysis of the Euler equation of the vane fluid machine. Schemes $S_{1}, S_{2}, S_{5}$, and $S_{6}$ for installing closed impellers have higher internal 


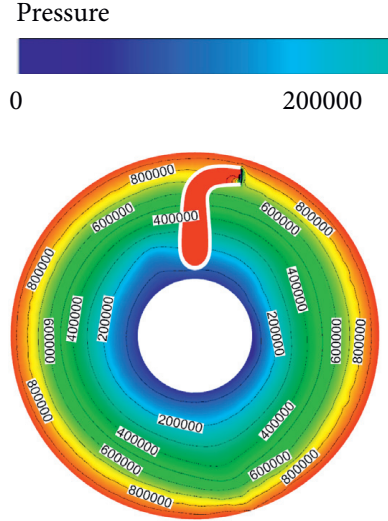

(a)

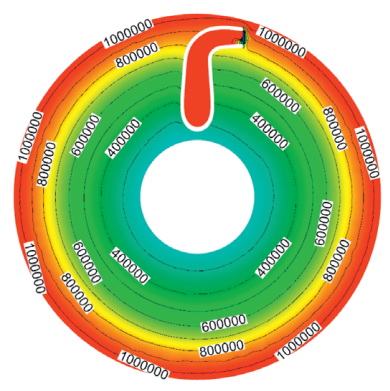

(e)

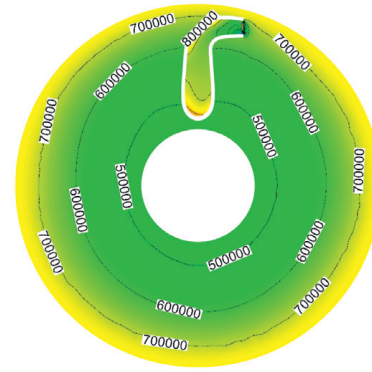

(b)

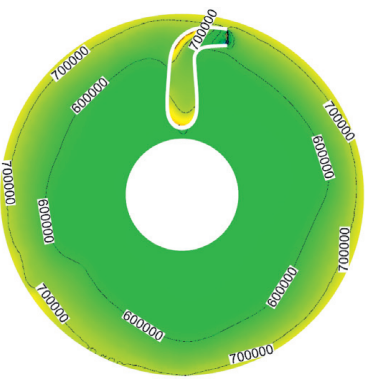

(f)

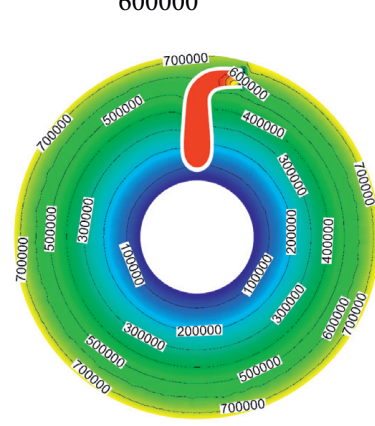

(c)

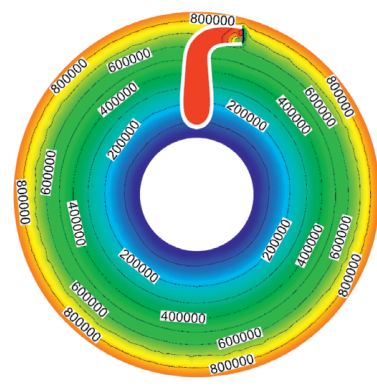

(g)
$\mathrm{Pa}$

$800000 \quad 1000000$

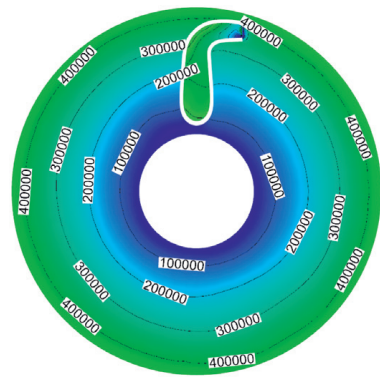

(d)

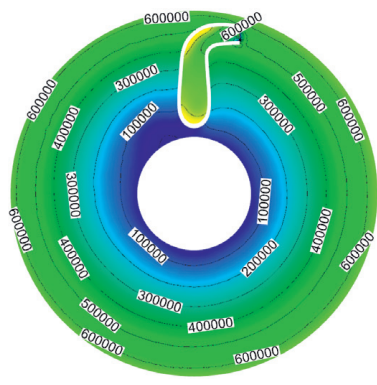

(h)

Figure 6: Pressure distribution at $Z_{1}$ characteristic section. (a) $S_{1}$. (b) $S_{2}$. (c) $S_{3}$. (d) $S_{4}$. (e) $S_{5}$. (f) $S_{6}$. (g) $S_{7}$. (h) $S_{8}$.

pressures than schemes $S_{3}, S_{4}, S_{7}$, and $S_{8}$, which use semiopen impellers in the same working conditions, indicating that the closed impellers are more effective in increasing the internal pressure of the Roto-Jet pump. The rotating casing rotation schemes $S_{1}, S_{3}, S_{5}$, and $S_{6}$ have a large pressure gradient in the chamber. Affected by the rotating casing effect, the liquid on the outer ring surface of the rotating casing is more obviously affected by the wall shear. The friction force is transferred to the central area of the flow to increase the internal pressure of the pump chamber, indicating that the rotating casing effect can improve the pressure distribution inside the rotating casing. As the rotating casings of schemes $S_{2}, S_{4}, S_{6}$, and $S_{8}$ do not rotate, the internal pressure of the collecting pipe is significantly lower than that of other spiral housing rotation schemes under the same working conditions. The internal pressure of the collecting pipe comes from the combination of the hydrostatic pressure and dynamic pressure inside the rotating casing. The nonrotation of the rotating casing causes the liquid circumferential shear velocity to decrease and the pressure inside the collecting pipe decreases. To accurately describe this problem, we further discuss the liquid shear velocity distribution inside the rotating casing.

\subsection{Liquid Shear Velocity Distribution in Rotating Casing.} We extract 150 pieces of tangential velocity distribution data equidistantly on the $O_{1}, O_{2}$, and $O_{3}$ curves in the $Z_{1}$ axis section. The calculation results are shown in Figure 7.
Figure 7 shows that the liquid velocity curve approximately forms concentric circles in the circumferential direction. In the same scheme, the larger the radius is, the greater the liquid tangential velocity is, which is consistent with the work principle of the centrifugal impeller. In Figure 7(a), the velocity curve shows a significant increase in the area of $335^{\circ} \sim 345^{\circ}$, which is located at the inlet of the collecting pipe. The decrease of the flow area causes the increase of the liquid shear velocity in this area. Affected by the liquid flow in the chamber by the collecting pipe, as shown in Figures 7(b) and $7(\mathrm{c})$, the speed of the upstream and wake regions of the collecting pipe fluctuates slightly, but the speed fluctuations in the upstream region $\left(335^{\circ} \sim 345^{\circ}\right)$ are more severe than those in the wake region $\left(15^{\circ} \sim 25^{\circ}\right)$. This result shows that the energy loss in the inlet area of the collecting pipe is relatively large, which is consistent with the research conclusion provided by Lu [18]. Schemes $S_{1}$, $S_{3}, S_{5}$, and $S_{7}$ have extremely close velocities on the curves $\mathrm{O}_{1}, \mathrm{O}_{2}$, and $\mathrm{O}_{3}$, with a deviation of less than $3 \%$, indicating that as long as the impeller and rotating casing continue to rotate synchronously, the liquid shear velocity at the same coordinate position in the chamber remains unchanged. The structure of the blade and impeller has a minimal effect on this value. When the casing is not rotating, the mean values of the liquid shear velocity in the chamber on the $\mathrm{O}_{1}, \mathrm{O}_{2}$, and $\mathrm{O}_{3}$ curves are schemes $S_{8}, S_{4}, S_{2}$, and $S_{6}$ in the order from large to small, with strong regularity. 


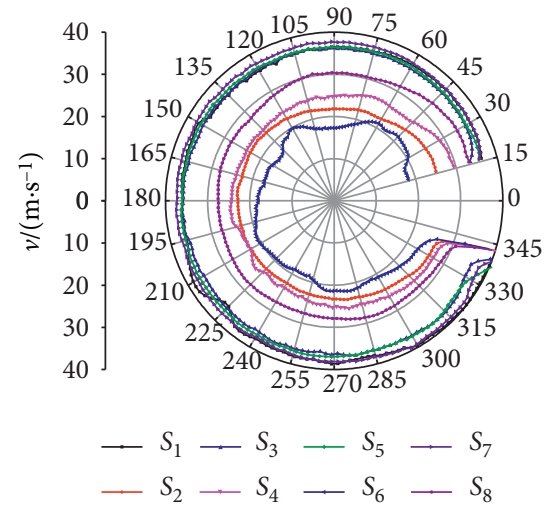

(a)

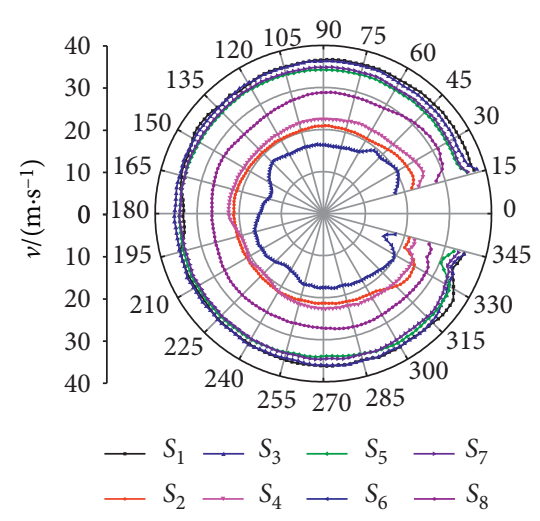

(b)

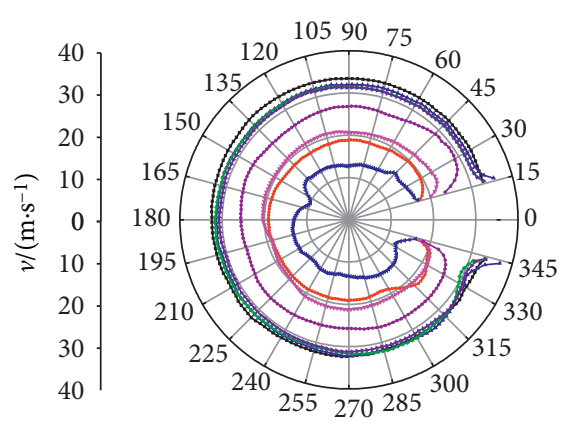

$-S_{1}-S_{3} \multimap S_{5} \multimap S_{7}$

$-S_{2}-S_{4}-S_{6}-S_{8}$

(c)

Figure 7: Velocity distribution of each curve at $Z_{1}$ characteristic section. (a) $O_{1}$ curve. (b) $O_{2}$ curve. (c) $O_{3}$ curve.

4.3. Uneven Coefficient of Liquid Shear Velocity. Borrowing the concepts of variance and turbulence intensity, we use the root mean square of the tangential velocity to measure the unevenness of the tangential velocity distribution along the inner circumference of the rotating casing. Thus, we can measure the influence of different schemes on the circumferential unevenness of the tangential velocity. The nonuniformity coefficient is expressed by the following formula [19]:

$$
\delta_{v}=\frac{\sqrt{\sum_{i=1}^{N}\left(v_{i}-\bar{v}\right)^{2}}}{N \bar{v}}
$$

where $v_{i}$ is the speed value at the constant measuring point, $\mathrm{m} / \mathrm{s} ; \bar{v}$ is the arithmetic mean of the speed at the constant measuring point, $\mathrm{m} / \mathrm{s} ; N$ is the number of constant measuring points, $N=150 . \delta_{v}$ is the circumferential nonuniformity coefficient of speed. The larger the nonuniformity coefficient, the greater the deviation of the circumferential speed. On the contrary, the deviation is small and close to the uniform distribution. Figure 8 shows the distribution of the uneven velocity coefficients on the curves $\mathrm{O}_{1} \sim \mathrm{O}_{7}$ of each scheme.

Figure 8 shows that, in the same scheme, the speed unevenness coefficient gradually increases along the radial direction. The larger the radius is, the greater the speed distribution unevenness coefficient is and the worse the speed uniformity is. This condition reflects the physical fact that the closer the interior of the rotating casing is to the axis, the faster the decrease of the peak tangential velocity is and the velocity tends to be uniform. In each plan, the arrangement of the same radius position and speed unevenness coefficient from small to large is $S_{1}, S_{5}, S_{3}, S_{7}, S_{8}, S_{4}, S_{6}$, and $S_{2}$. To summarise its regularity, the rotating casing effect can reduce the uneven coefficient of liquid velocity, improve the distribution of tangential velocity, and dominate all factors. The second influencing factor is the front cover. When the rotating casing rotates with the impeller, the front cover promotes the uniformity of the shear velocity. When the rotating casing is fixed, the front cover can inhibit the uniformisation of the liquid shear velocity in the chamber;

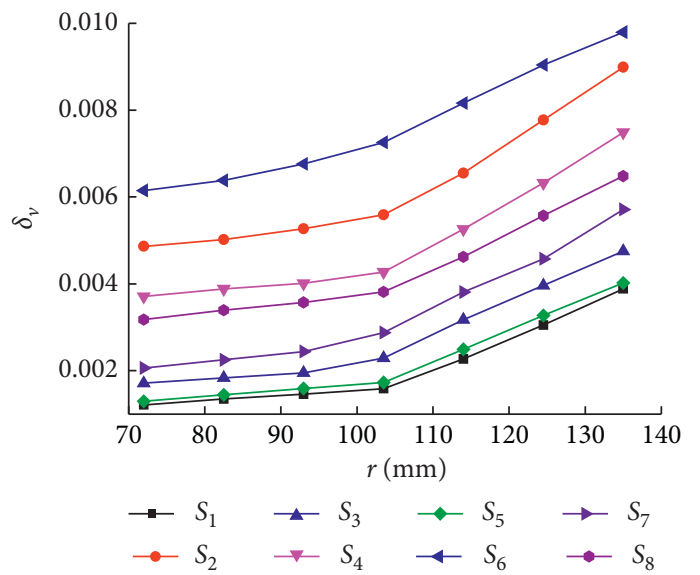

FIgURE 8: Coefficient of uneven tangential velocity distributed along radial direction.

the blade type has a weak effect on the circumferential shear velocity distribution.

4.4. Vortex Distribution inside Rotating Casing. Due to the existence of the collecting pipe, the interior of the rotating casing is always accompanied by a strong vortex, which inevitably affects the internal flow of the pump. Therefore, analysing the internal vortex structure of the rotating casing under different schemes is necessary. The $Q$ criterion [20] is the most common vortex identification method based on the velocity gradient tensor. It reflects a balance between the rotation and deformation of a fluid cluster in the flow field. $Q>0$ reflects the dominant position of rotation in the flow. The greater the $Q$ value, the greater the vortex intensity. Figure 9 shows the variation of the vortex cores with the same $Q$ value inside the rotating casings of schemes $S_{1} \sim S_{8}$ under rated conditions. Different colours indicate the velocity distribution area in the vortex core.

In the flow around the collecting pipe, the fluid touches the bluff body and separates, the flow area shrinks, and the flow velocity increases, thereby resulting in differential pressure resistance and causing boundary layer separation 


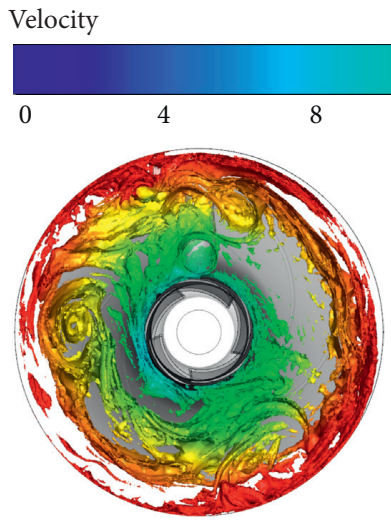

(a)

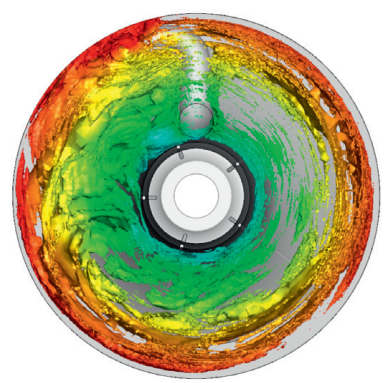

(e)

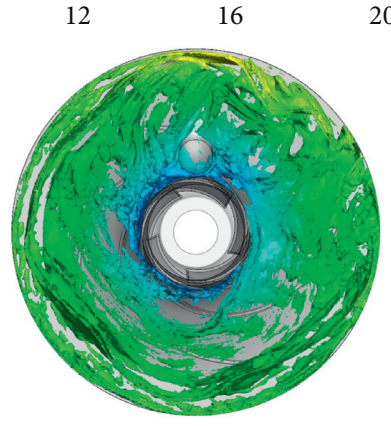

(b)

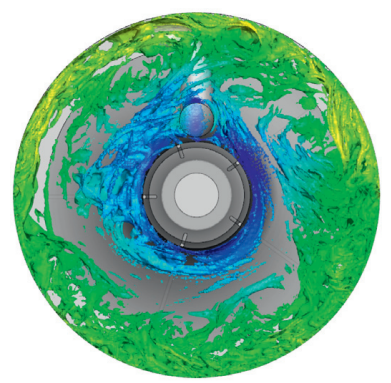

(f)

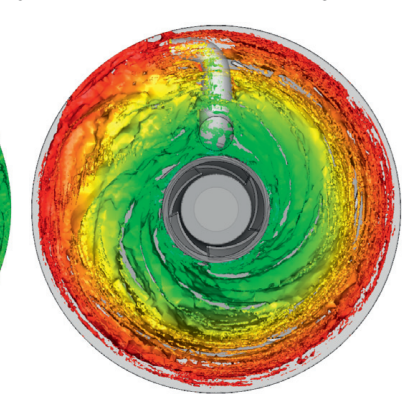

(c)

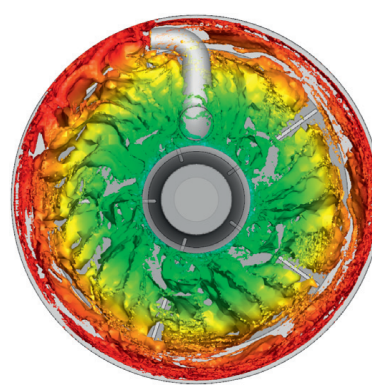

(g)

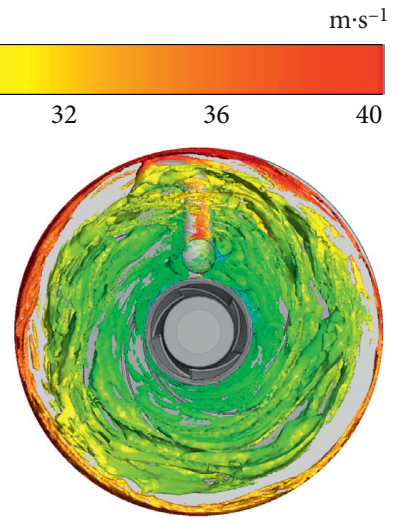

(d)

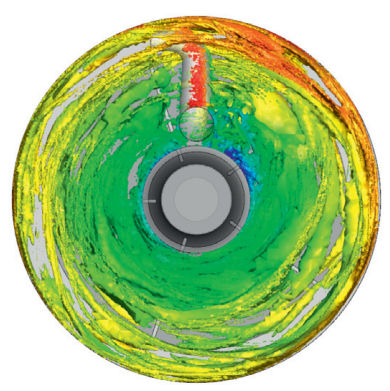

(h)

FiguRE 9: Regional vortex core distribution of different schemes with same $Q$ values $=10000$. (a) $S_{1}$. (b) $S_{2}$. (c) $S_{3}$. (d) $S_{4}$. (e) $S_{5}$. (f) $S_{6}$. (g) $S_{7}$. (h) $S_{8}$.

and vortex flow. According to Figure 9, the change of the $Q$ value shows that schemes $S_{1}$ and $S_{5}, S_{2}$ and $S_{6}, S_{3}$ and $S_{7}$, and $S_{4}$ and $S_{8}$ have similar vortex core distribution structures, indicating that the blade structure has a weaker influence on the vortex core. $S_{1}$ and $S_{5}$ did not have a large wake vortex but a clear front wing vortex. The fluid gathered at the inlet of the collecting pipe and collided with the pipe, which is the main reason for the formation of the front wing vortex. The split flow at the inlet of the collecting pipe leads to a relatively small decrease in the flow velocity around the flow. The vortex extends backward from the surface of the collecting pipe after touching the collecting pipe. The high-speed rotation of the front cover of the runner and rotating casing causes the wake vortex to tear quickly, the wake vortex spreads into small fragments, and a blank space exists at the end of the collecting pipe, which further shows that the energy loss of the jet pump is mainly concentrated in the inlet area of the collecting pipe. In schemes $S_{2}$ and $S_{6}$, the high-speed impeller in the vortex core is no longer obvious. On the contrary, the vortex is mainly concentrated on the outside of the rotating casing. When the rotating casing does not rotate, the relative linear velocity of the liquid and the outer ring surface of the rotating casing are quite different. The two frictions create vortices and the dynamic processes such as generation, dissipation, and redistribution of pulsating energy near the wall are more dynamic; the vigorous vortex in the centre area is small. In schemes $S_{3}$ and $S_{7}$, the distribution of vortices is obvious. The half-open impeller accelerates the speed of the exchange flow and uniform flow of energy inside the impeller. This trend is exacerbated by the synchronous rotation of the rotating casing. In schemes $S_{4}$ and $S_{8}$, strong vortices occur in the flow centre area and outer annular surface of the rotating casing, and the vortex core flocculent structure generated by the suction surface of the impeller fills the interior of the rotating casing. We found by comparison that the size, shape, and position of the vortex centre of the vortex are constantly changing with the different schemes. The distribution of the vortex core under each scheme is different. There is a more serious effect of the front cover and rotating casing on the vortex core and the influence of the nuclear structure is weak.

4.5. Distribution of Liquid Rotational Angular Velocity. Some researchers [21, 22] believe that, in an ordinary centrifugal pump, the average rotational angular velocity of the liquid in the chamber is half of the rotational angular velocity of the impeller and the liquid conforms to the law of rigid motion. Zhang and Tang [23, 24] pointed out that the liquid rotation in the Roto-Jet pump chamber conforms to the rigid motion law and the liquid rotation angular velocity is $78 \%$ of the rotating casing angular velocity. For this reason, this study analyses the liquid rotation angular velocity of each scheme in the $Z_{1}$ axis section. The relationship between the liquid rotation angular velocity and radius of the different schemes is presented in Figure 10. 


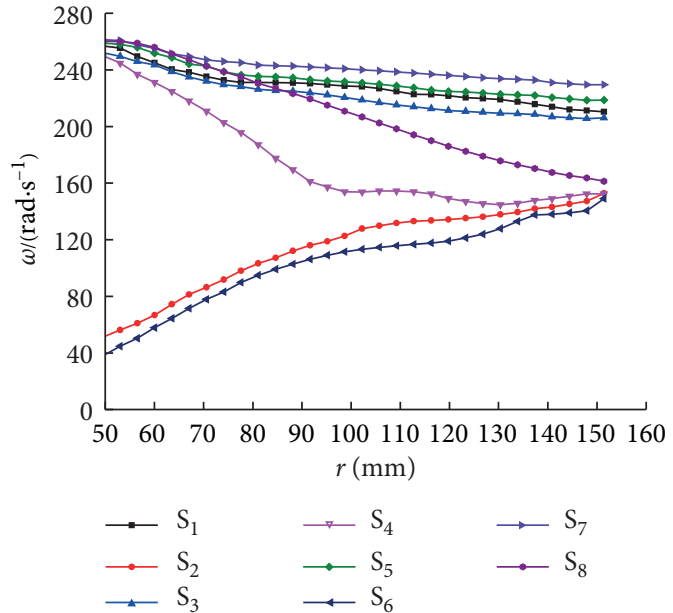

Figure 10: Distribution of liquid rotation coefficient in pump chamber.

When the impeller and rotating casing rotate synchronously, the discrete points of schemes $S_{1}, S_{5}, S_{3}$, and $S_{7}$ are similar to a straight line and the liquid conforms to the law of rigid body motion. The average liquid rotational angular velocities for schemes $S_{1}, S_{5}, S_{3}$, and $S_{7}$ are 228.4, 221.9, 233.2, and $241.5 \mathrm{rad} / \mathrm{s}$. The rotation speeds of the rotating casing for schemes $S_{1}, S_{5}, S_{3}$, and $S_{7}$ are $75.3 \%, 73.1 \%, 76.8 \%$, and $79.6 \%$ of $303.5 \mathrm{rad} / \mathrm{s}$, which are close to the conclusions reported by Zhang and Tang $[23,24]$. When the rotating casing is not rotating, the liquid rotation angular velocity is greatly affected by the front cover plate. The specific performance is that the liquid rotation angular velocity of the front cover plates $S_{2}$ and $S_{6}$ gradually increases in the radial direction. At this time, the liquid rotation in the rotating casing comes from the wall friction of the cover plate and momentum transport of the liquid at the impeller outlet. When the radius is small, the wall friction is dominant and the liquid rotation angular velocity is small. When the radius is large, the liquid momentum transport at the impeller outlet dominates and the liquid rotation angular velocity increases. Solutions $S_{4}$ and $S_{5}$ without the front cover plate gradually reduce the liquid rotation angular velocity along the radial direction, which indicates that the liquid rotation in the rotating casing is mainly due to the blade constraint. When the radius is small, the blade circumferential area accounts for a large proportion, which is strong for liquid. When the rotational angular velocity and radius are large, the area of the blade in the circumferential direction is small, the liquid is weakly constrained, and the liquid rotational angular velocity gradually decreases.

4.6. Turbulent Energy Distribution in Impeller. The basic parameter that describes turbulence is turbulent kinetic energy (TKE), an important parameter that characterises the intensity of turbulent motion and maintains the development and attenuation of turbulence $[25,26]$. Its increase causes an increase in liquid energy loss and a decrease in pump head and efficiency, increasing rotatory machinery failure $[27,28]$. A flow field with low turbulence is often relatively stable. The TKE distribution of the $Z_{2}$ axis section under the eight schemes is shown in Figure 11.

Figure 11 shows that the TKE distribution of all the schemes is basically symmetrical about the axis. The backward-curved closed impeller has better liquid confinement and high liquid flow stability. The turbulent flow energy of schemes $S_{1}$ and $S_{2}$ is evenly distributed. Owing to the use of straight blades in schemes $S_{5}$ and $S_{6}$, the area with greater turbulence energy is concentrated in the impeller inlet. Schemes $S_{3}, S_{4}, S_{7}$, and $S_{8}$ use semiopen impellers, which have poor liquid confinement, serious liquid recirculation and swirling, high turbulent flow energy, and large energy loss. The mean values of the turbulent flow energy per unit volume of the liquid inside the impellers of schemes $S_{1} \sim S_{8}$ are $0.11,0.13,0.57,0.98,0.49,0.92,1.58$, and $2.46 \mathrm{~m}^{2} \cdot \mathrm{s}^{-2}$. We judge that the internal flow stability of the $S_{1}$ impeller is high and the performance is improved.

4.7. Internal Flow Analysis of Collecting Pipe. The collecting pipe consists of suction, diffusion, and stabilisation sections. The liquid flowing out of the impeller enters the diffusion section through the suction section. The function of the diffusion section is to convert the speed energy of the liquid into pressure energy so that the liquid being transported can be in the pipeline and the energy loss is minimal. Therefore, studying the relationship between the pressure and velocity of the liquid inside the collecting pipe and the parameters of the Roto-Jet pump is important. Schemes $S_{1} \sim S_{8}$ in the collecting pipe and pressure distribution along the central axis of the collecting pipe are shown in Figure 12.

Figure 12(a) shows that the inlet pressure of the collecting pipe basically corresponds to the pressure inside the rotating casing. The higher the pressure inside the rotating casing, the higher the inlet pressure of the collecting pipe. Due to the rapid expansion and distortion of its pipe diameter, the pressure curve of the collecting pipe rises significantly when $l<40 \mathrm{~mm}$ and the pressure diffusion capacity is strong. Thereafter, the pressure curve shows a steady upward trend and the movement state is relatively stable. The shape of the internal pressure curve of the collecting pipe under each scheme is consistent, which shows that the rotating casing and impeller structure have a certain influence on the internal pressure of the collecting pipe but a minimal effect on the pressure diffusion capacity of the collecting pipe. Figure 12(b) shows that the velocity curve continues to decrease from the inlet to the outlet of the collecting pipe. The velocity decreases significantly in the range of $l<40 \mathrm{~mm}$ and tends to be stable in the range of $l>120 \mathrm{~mm}$. The difference in the internal velocity distribution of the collecting pipe of the various schemes is small and negligible, which means that when the flow is constant and the structure of the collecting pipe is unique, the impeller structure and rotating casing have no effect on the internal velocity distribution of the collecting pipe. Based on a certain pressure-bearing capacity of the collecting pipe, its overcurrent and expansion capacity should be prioritised. Selecting a reasonable length of the expansion section can 


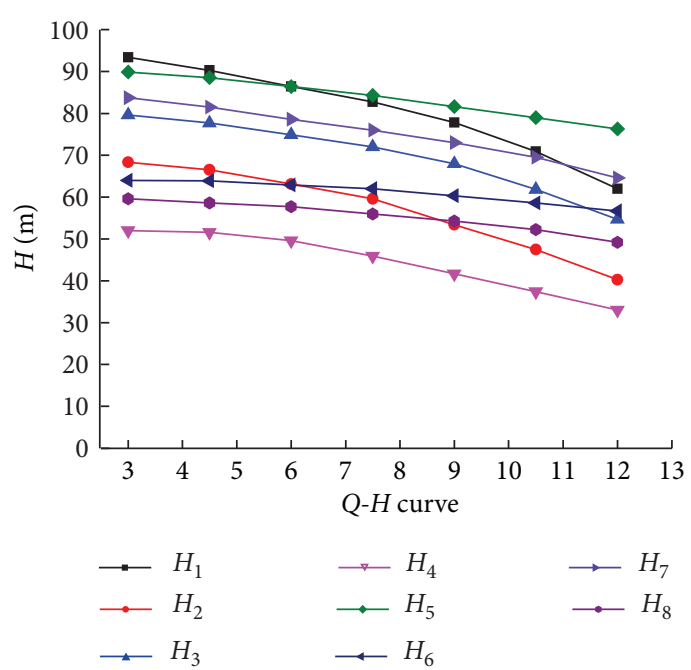

(a)

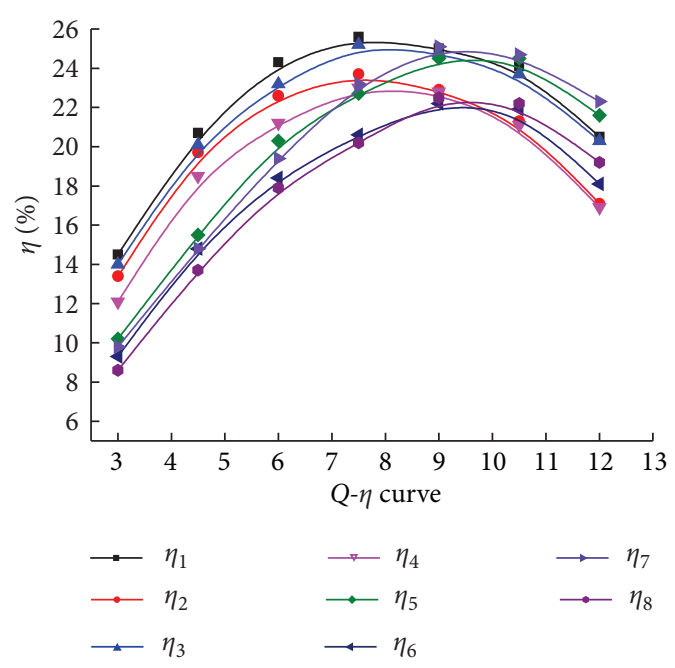

(b)

Figure 13: Performance curve. (a) $Q-H$ curve. (b) $Q-\eta$ curve.

backward curved blade and stable head curve of the straight blade. The deviation of the head of the rated flow point and design value of $80 \mathrm{~m}$ is $3.4 \%,-25.5 \%,-10.0 \%,-42.6 \%, 5.4 \%$, $-22.5 \%,-5.1 \%$, and $-30.7 \%$, respectively. Only schemes $S_{1}$ and $S_{5}$ meet the requirements. Due to the influence of the blade type, scheme $S_{1}$ has a higher head under small flow conditions and the head decreases significantly with the increase in flow, while the head under large flow conditions is lower. Scheme $S_{5}$ has a gentle head curve and stable performance compared with scheme $S_{1}$, and scheme $S_{5}$ has a higher head in large flow conditions and a more powerful impeller.

For the $Q-\eta$ curve, the high-efficiency zone of schemes $S_{1} \sim S_{4}$ is wider and the highest efficiency point is near the rated flow. The high-efficiency zone of schemes $S_{5} \sim S_{8}$ is narrow and the high-efficiency zone moves backward near $1.2 Q_{0}$. This situation is related to the displacement of the blade inlet. The back-curved impeller inlet is seriously crowded, which restricts the large flow conditions. In schemes $S_{1} \sim S_{8}$, the deviations of the rated flow point efficiency and design value $25 \%$ are $2.4 \%,-5.2 \%, 0.8 \%,-8.0 \%$, $-2.0 \%,-11.2 \%, 0.4 \%$, and $-10.0 \%$. Scheme 1 is the most efficient.

\section{Extremely Poor Analysis of Test Results}

The data of the highest efficiency point on each test plan are shown in Table 3.

Using the results in Table 3, we can conclude that the highest efficiency point flow of the Roto-Jet pumps of schemes $S_{1} \sim S_{4}$ is in good agreement with the design working conditions and the highest efficiency point of Roto-Jet pumps of schemes $S_{5} \sim S_{8}$ is biased toward large flow working conditions. Table 4 presents a range analysis table for flow, head, and efficiency at the highest efficiency point. When determining the level of each factor, we can compare the sizes of indexes $K_{1}$ and $K_{2}$. In this test, the higher the flow rate, head, and efficiency index of the highest efficiency point
TABLe 3: Results of the highest efficiency point test.

\begin{tabular}{lccc}
\hline Test scheme & $Q\left(\mathrm{~m}^{3} \cdot \mathrm{h}^{-1}\right)$ & $H(\mathrm{~m})$ & $\eta(\%)$ \\
\hline$S_{1}$ & 7.5 & 82.7 & 25.6 \\
$S_{2}$ & 7.5 & 59.5 & 23.7 \\
$S_{3}$ & 7.5 & 72.1 & 25.4 \\
$S_{4}$ & 7.5 & 45.9 & 23.0 \\
$S_{5}$ & 9 & 81.6 & 24.5 \\
$S_{6}$ & 9 & 60.3 & 22.2 \\
$S_{7}$ & 9 & 73.0 & 25.1 \\
$S_{8}$ & 9 & 54.8 & 22.5 \\
\hline
\end{tabular}

TABLE 4: Flow and head analysis at the highest efficiency point.

\begin{tabular}{ccccc}
\hline & Index & Blade type & Front cover & Rotating casing \\
\hline & $K_{1}$ & 30.0 & 33.0 & 33.0 \\
$Q$ & $K_{2}$ & 36.0 & 33.0 & 33.0 \\
& $K_{1}$ & 7.5 & 8.3 & 8.3 \\
& $K_{2}$ & 9.0 & 8.3 & 8.3 \\
& $R$ & 1.5 & 0.0 & 0.0 \\
\hline \multirow{4}{*}{$H$} & $K_{1}$ & 260.2 & 284.1 & 309.4 \\
& $K_{2}$ & 269.7 & 245.8 & 220.5 \\
& $K_{1}$ & 65.1 & 71.0 & 77.4 \\
& $K_{2}$ & 67.4 & 61.5 & 55.1 \\
& $R$ & 2.3 & 9.5 & 22.3 \\
\hline \multirow{4}{*}{$\eta$} & $K_{1}$ & 97.6 & 96.0 & 100.6 \\
& $K_{2}$ & 94.3 & 95.9 & 91.3 \\
& $K_{1}$ & 24.4 & 24.0 & 25.2 \\
& $K_{2}$ & 23.6 & 23.9 & 22.8 \\
& $R$ & 0.8 & 0.1 & 2.4 \\
\hline
\end{tabular}

are, the better the performance of the Roto-Jet pump is, so the optimal combination (largest combination of factors) should be selected.

According to the size of the range, the primary and secondary orders of the influence of various factors on the flow rate of the highest efficiency point are blade type, front 
cover, and rotating casing. Taking $A_{2}, B_{1} / B_{2}$, and $C_{1} / C_{2}$ according to the size of the index value is the optimal solution for the highest efficiency point flow. The primary and secondary orders of the impact on the head of the highest efficiency point are the rotating casing, front cover, and blade type. According to the size of the index value, we take $A_{2}, B_{1}$, and $C_{1}$ as the optimal solution for the head of the highest efficiency point. The primary and secondary orders are the rotating casing, blade type, and front cover. According to the index value, we take $A_{1}, B_{1}$, and $C_{1}$ as the best solution for the highest efficiency point. Through a comprehensive comparison of the optimal conditions of each index, the blade type has a decisive effect on the flow rate of the optimal efficiency point of the Roto-Jet pump and the rotating casing has the most obvious influence on the head and efficiency of the Roto-Jet pump.

\section{Conclusion}

(1) The rotating casing effect has multiple functions of reducing the friction loss of the disc, improving the internal pressure distribution of the rotating casing, and increasing the pump head. The internal pressure and tangential velocity of the rotating casing increase with the radius increased. The pressure and velocity of the liquid fluctuate in the upstream and wake regions of the collecting pipe, but the pressure and velocity fluctuations in the upstream region are more intense and the energy loss is concentrated in the upstream area. The greater the internal pressure of the rotating casing is and the higher the internal pressure of the collecting pipe is, the smaller the difference in the internal velocity distribution of the collecting pipe is, which can be ignored. We recommend that the flow and pressure expansion capabilities of the collecting pipe be prioritised on the basis of a certain pressure-bearing capacity.

(2) As long as the impeller and rotating casing continue to rotate synchronously, the liquid shear velocity at the same coordinate position of each scheme remains unchanged. The liquid rotation angular velocity is approximately $75 \%$ of the rotating casing speed and the liquid conforms to the rigid motion law. In the same scheme, the velocity nonuniformity coefficient gradually increases along the radial direction, which reflects the physical fact that the closer the interior of the rotating casing is to the axis, the faster the decrease of the peak tangential velocity is and the velocity tends to be uniform.

(3) The distribution of turbulent kinetic energy in the $Z_{2}$ axis section of all schemes is symmetrical on the axis, and the backward-curved closed impeller has good liquid confinement and high liquid-flow stability. The size, shape, and position of the vortex core in the rotating casing constantly change with different schemes. The distribution of vortex cores in the rotating casing varies under each scheme. The front cover and rotating casing have a strong effect on the vortex core and the blade type has a weak influence.

(4) The range analysis of the test results shows that the blade type has a decisive effect on the flow rate of the optimal efficiency point of the Roto-Jet pump and the rotating casing has the most obvious influence on the head and efficiency. The optimal solution of the Roto-Jet pump uses the rotary casing and enclosed impeller to rotate synchronously. The advantages and disadvantages of each blade type can be determined according to the situation.

\section{Data Availability}

The curve data used to support the findings of this study are available from the corresponding author upon request.

\section{Conflicts of Interest}

The authors declare that they have no conflicts of interest.

\section{Acknowledgments}

This project was supported by the National Natural Science Foundation of China (Grant no. 51969014).

\section{References}

[1] S. Osborn, "The Roto-Jet pump: 25 years new," World Pumps, vol. 1996, no. 363, pp. 32-36, 1996.

[2] J. Yang, X. Qi, and X. Ma, "Efficiency analysis of roto-jet pump and hydraulic design of collecting pipe," Journal of Lanzhou University of Technology, vol. 21, no. 2, pp. 33-36, 1995.

[3] Y. Tanasawa and Y. Miyasaka, "A study on the pitot pump:1st report. On the characteristics of pressure pumps (lectures in commemoration of retirement of prof. Miyagi.)," Journal of the Society of Mechanical Engineers, vol. 51, no. 358, pp. 265-266, 1948.

[4] Y. Tanasawa and Y. Miyasaka, "A study on the pitot pump (1st report, on the characteristics of pressure pumps)," Transactions of the Japan Society of Mechanical Engineers, vol. 15, no. 51, pp. 39-43, 1950.

[5] J. Yang, "Theoretical head and impeller optimization design of roto-jet pump," Journal of Lanzhou University of Technology, vol. 21, no. 1, pp. 26-30, 1995.

[6] Y. Zhu, C. Kang, and M. A. O. Ning, "Influence of the collecting pipe and the inner guide vane on roto-jet pump performance international," Journal of Fluid Dynamics, vol. 4, no. 3, pp. 33-42, 2016.

[7] Y. Wang, C. Chen, Y. Chang-Ming et al., "Research of internal flow with in the impeller of roto-jet pump," Journal of Xihua University:Natural Science Edition, vol. 9, no. 23, pp. 194-196, 2004.

[8] Y. Liu, W. Chen, R. Liang et al., "Structure optimization and flow numerical simulation in rotor chamber of roto-jet pump," Journal of Drainage and Irrigation Machinery Engineering, vol. 1, no. 1, pp. 26-30, 2015.

[9] K. Komaki, T. Kanemoto, K. Sagara et al., "Effect of the collecting pipe tube profile on pitot pump performances," IOP Conference Series Materials Science and Engineering, vol. 52, no. 3, 2013. 
[10] K. Komaki, T. Kanemoto, and K. Sagara, "Performances and Rotating Flows of Roto-Jet Pump," Open Journal of Fluid Dynamics, vol. 2, no. 4, pp. 375-379, 2013.

[11] K. Sagara, K. Furumaki, T. Kanemoto, and T. Umekage, "129 prediction of pitot pump internal flow using CFD," Proceedings of Kyushu Chapter of the Japan Society of Mechanical Engineers, vol. 67, pp. 129-1-129-2, 2014.

[12] T. Hattori, "Structure of the Pitot Tube on the Pitot Pump," Japanese Unexamined Patent Application Publication No. Hei 9-324785, 1997.

[13] A. Kato, "Pitot Pump," Japanese Unexamined Patent Application Publication No. Hei 6-241184, 1994.

[14] J. Yang and X. Qi, "Discussion on efficiency of roto-jet pump and hydraulic design method of collecting pipe," Chemical Machinery, vol. 45, no. 2, pp. 29-31, 1996.

[15] Y. Yang, L. Zhou, W. Shi, Z. He, Y. Han, and Y. Xiao, "Interstage difference of pressure pulsation in a three-stage electrical submersible pump," Journal of Petroleum Science and Engineering, vol. 196, Article ID 107653, 2020.

[16] L. Zhou, C. Han, L. Bai, W. Li, M. El-Emam, and W. Shi, "CFD-DEM bidirectional coupling simulation and experimental investigation of particle ejections and energy conversion in a spouted bed," Energy, vol. 211, Article ID 118672, 2020.

[17] S. Yuan, W. Shi, and H. Liu, Pump Theory and Technology, China Machine Press, Beijing, China, 2014.

[18] J. Lu, Flow Simulation and Analysis for the Rotor Chamber and the Collecting Tube of a Rotor-Jet pump, Ph.D. thesis, Jiangsu University, Zhenjiang, China, 2017.

[19] W. Li and Y. Zhang, Theory and Application of Centrifugal Oil Pump, Jiangsu University Press, Zhenjiang, China, 2016.

[20] P. Chakraborty, S. Balachandar, and R. J. Adrian, "On the relationships between local vortex identification schemes," Journal of Fluid Mechanics, vol. 535, pp. 189-214, 2005.

[21] Z. Liu, D. Wang, W. Hou et al., "Experiment and calculation of fluid pressure in pump chamber and balance chamber of centrifugal pump," Transactions of the Chinese Society for Agricultural Machinery, vol. 47, no. 8, pp. 42-47, 2016.

[22] W. Dong and W. Chu, "Analysis of flow characteristics and disc friction loss in balance chamber of centrifugal pump impeller," Transactions of the Chinese Society for Agricultural Machinery, vol. 47, no. 4, pp. 29-35, 2016.

[23] H. Zhang, Characteristic of the start-up and analysis about inside flow field of the roto-jet pump, Ph.D. thesis, Lanzhou University of Technology, Lanzhou, China, 2009.

[24] L. Tang, .Calculation and Analysis about inside Flow Field and Performance Prediction for Roto-Jet pump, Ph.D. thesis, Lanzhou University of Technology, Lanzhou, China, 2008.

[25] Z. Zhang, G. Cui, C. Xu et al., Theory and Modeling of Turbulence, Tsinghua University Press, Beijing, China, 2nd edition, 2017.

[26] H. Quan, J. Cheng, Y. Guo, L. Kang, and G. Peng, "Influence of screw centrifugal inducer on internal flow structure of vortex pump," ASME Journal of Fluids Engineering, vol. 142, no. 9, Article ID 091203, 2020.

[27] S. Tang, S. Yuan, and Y. Zhu, "Deep learning-based intelligent fault diagnosis methods toward rotating machinery," IEEE Access, vol. 8, no. 1, pp. 9335-9346, 2020.

[28] S. Tang, S. Yuan, and Y. Zhu, "Convolutional neural network in intelligent fault diagnosis toward rotatory machinery," IEEE Access, vol. 8, no. 1, pp. 86510-86519, 2020.

[29] H. Quan, G. U. O. Ying, L. I. Rennian et al., "Optimization design and experimental study of vortex pump based on orthogonal test," Science Progress, vol. 20, pp. 1-20, 2019.
[30] H. Quan, C. H. A. I. Yi, L. I. Rennian et al., "Numerical simulation and experiment for study on internal flow pattern of vortex pump," Engineering Computation, vol. 36, pp. 1579-1596, 2019.

[31] H. Quan, C. H. A. I. Yi, L. I. Rennian et al., "Numerical simulation and experiment for study on internal flow pattern of vortex pump," Engineering Computation, vol. 36, pp. 1579-1596, 2019. 